\title{
Results of Total Sample
}

Tab. 1 Sample characteristics of total sample

\begin{tabular}{ll}
\hline sample size & 730 \\
age in years (mean, standard deviation) & $37.7(13.1)$ \\
gender (female) $(\%)$ & 77.7 \\
marital status (\%) & \\
$\quad$ single & 47.5 \\
$\quad$ in partnership & 26.2 \\
$\quad$ others & 26.3 \\
level of education (\%) & \\
$\quad$ <12 years of school education & 39.0 \\
$\quad \geq 12$ years of school education & 61.0 \\
current type of mental health care (\%) & \\
$\quad$ outpatient & 66.2 \\
$\quad$ inpatient & 14.9 \\
$\quad$ not applicable & 18.9 \\
current severity of depression (mean, standard deviation) & $14.7(6.0)$ \\
stereotype awareness (mean, standard deviation) & $51.1(17.4)$ \\
personal agreement (mean, standard deviation) & $24.3(11.8)$ \\
self-concurrence (mean, standard deviation) & $26.9(13.9)$ \\
self-esteem (mean, standard deviation) & $13.4(7.1)$ \\
\hline
\end{tabular}


Tab. 2 Bivariate nonparametric Spearman correlations of model variables of total sample

\begin{tabular}{|c|c|c|c|c|c|c|c|}
\hline & 1 & 2 & 3 & 4 & 5 & 6 & 7 \\
\hline 1 gender & -- & & & & & & \\
\hline 2 age & $0.081 *$ & -- & & & & & \\
\hline 3 depression & -0.031 & $-0.091 *$ & -- & & & & \\
\hline 4 awareness & $-0.089 *$ & -0.071 & $0.166^{* * *}$ & -- & & & \\
\hline 5 agreement & $0.078 *$ & $-0.078^{*}$ & $0.176^{* * *}$ & $0.186 * * *$ & -- & & \\
\hline 6 self-concurrence & $0.079 *$ & $-0.155 * * *$ & $0.447 * * *$ & $0.154 * * *$ & $0.532 * * *$ & -- & \\
\hline 7 self-esteem & $0.087 *$ & $0.263 * * *$ & $-0.603 * * *$ & $-0.205 * * *$ & $-0.201 * * *$ & $-0.465 * * *$ & -- \\
\hline
\end{tabular}

depression=current level of depression; awareness=stereotype awareness; agreement=personal agreement; $* * * \mathrm{p}<0.001 ; * * \mathrm{p}<0.01 ; * \mathrm{p}<0.05$ 

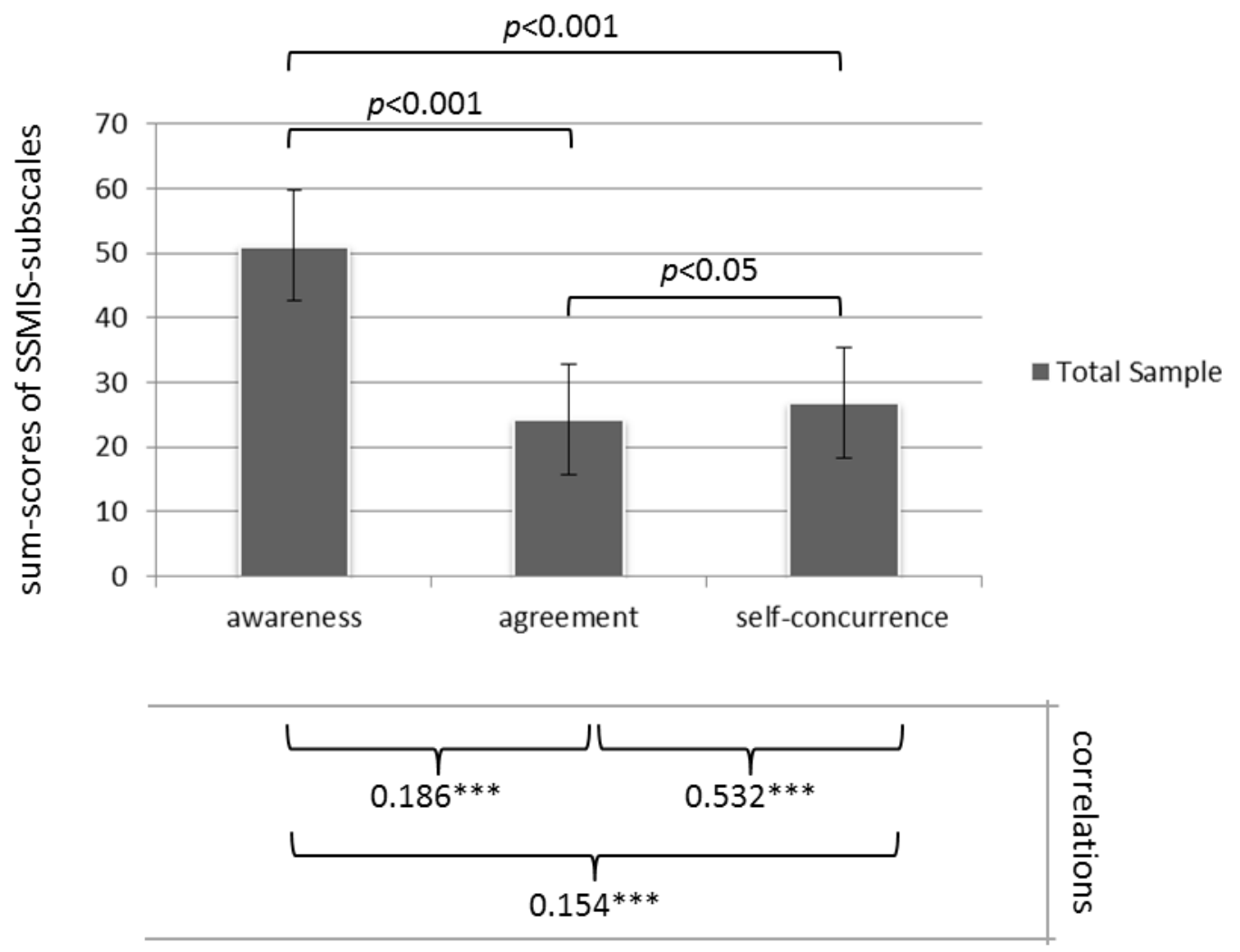

Fig 2. Summed scores and direct associations of stigma attitudes in total sample.

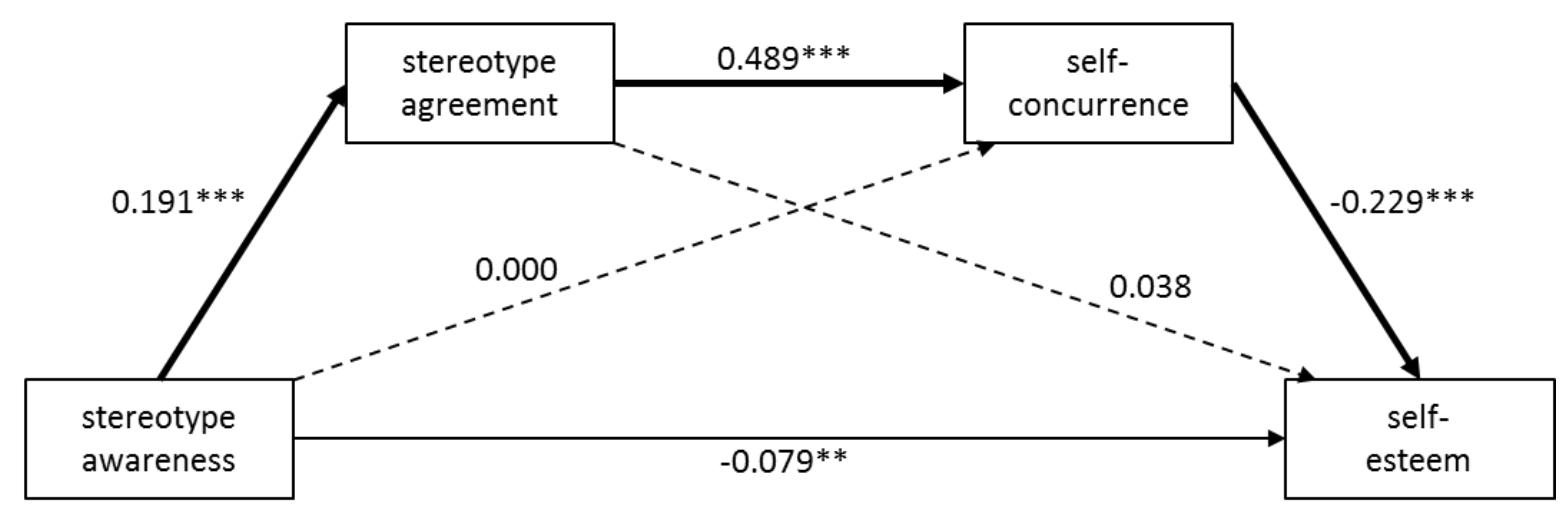

Fig 3. Serial mediation model of total sample. Solid lines indicate significant direct paths. Thick solid lines indicate significant indirect paths. Dotted lines represent non-significant direct paths. Standardized coefficients are presented. ${ }^{* * *} \mathrm{p}<0.001 ; * * \mathrm{p}<0.01 ; * \mathrm{p}<0.05$. 



\section{Results of Serial Mediation Model of Total Sample}

PROCESS Procedure for SPSS Version 3.00 (Written by Andrew F. Hayes. Ph.D. www.afhayes.com)

Documentation available in Hayes (2018). www.guilford.com/p/hayes3

Model: 6

Y: $\quad$ self-esteem

$\mathrm{X}: \quad$ stereotype awareness

M1: stereotype agreement

M2: self-concurrence

Covariates: age gender depression

Sample Size: 730

\section{Direct effects}

Outcome variable: stereotype agreement

Model summary

$\begin{array}{lllllll}\mathrm{R} & \mathrm{R}^{2} & \mathrm{MSE} & \mathrm{F} & \mathrm{df} 1 & \mathrm{df2} & p \\ 0.2728 & 0.0744 & 0.9294 & 14.5786 & 4.0000 & 725.0000 & 0.0000\end{array}$

Model

constant

stereotype awareness

age

gender

depression

coeff
0.0000
0.1905
-0.0413
0.0918
0.1408

$\mathrm{se}$
0.0357
0.0364
0.0361
0.0359
0.0363

$\mathrm{t}$
0.0000
5.2345

$\begin{array}{rr}p & \text { LLCI } \\ 1.0000 & -0.0701 \\ 0.0000 & 0.1190 \\ 0.2527 & -0.1120 \\ 0.0107 & 0.0214 \\ 0.0001 & 0.0695\end{array}$

ULCI 0.0701

Outcome variable: self-concurrence

Model summary

$\mathrm{R}^{2}$

0.658

0.4331

MSE

0.5700

F

110.6199

df1

5.0000

df2

724.0000

Model

0.2121

constant

stereotype awareness

stereotype agreement

age

gender

depression

$\begin{array}{rr}\text { coeff } & \text { se } \\ 0.0000 & 0.0279 \\ 0.0002 & 0.0290 \\ 0.4887 & 0.0291 \\ -0.1001 & 0.0283 \\ 0.0579 & 0.0282 \\ 0.3311 & 0.0287\end{array}$

$0.0000^{\mathrm{t}}$

0.0074

16.8026

$-3.5418$

2.0507

11.5177

$p$
1.0000
0.9941
0.0000
0.0004
0.0407
0.0000

LLCI

$-0.0549$

$-0.0568$

0.4316

$-0.1556$

0.0025

0.2747

Outcome variable: self-esteem

Model summary

$\mathrm{R} \quad \mathrm{R}^{2}$

$0.6770 \quad 0.4583$

MSE

0.5455

F

df1

6.0000

df2

723.0000

$p$

Model

constant

stereotype awareness

stereotype agreement

self-concurrence

coeff
0.0000
-0.0792
0.0383
-0.2285
0.1651
0.0589
-0.4928

se
0.0273

0.0284

0.0335

0.0364

0.0279

0.0277

gender

depression

0.0306

$0.0000^{\mathrm{t}}$

$-2.7894$

1.1429

$-6.2840$

5.9209

2.2169

$-16.1098$

$p$
1.0000
0.0054
0.2535
0.0000
0.0000
0.0338
0.0000

LLCI

$-0.0537$

$-0.1350$

$-0.0275$

$-0.2998$

0.1130

0.0045

$-0.5529$

ULCI

0.0549

0.0572

0.5458

$-0.0446$

0.1134

0.3878

\section{Total effect}

Outcome variable: self-esteem

Model summary

$\begin{array}{lll}\mathrm{R} & \mathrm{R}^{2} & \mathrm{MSE} \\ 0.6509 & 0.4237 & 0.5787\end{array}$

MSE
0.5787

F

133.2680

df1
4.0000

df2

725.0000

0.0000

Model

constant

stereotype awareness

coeff

se
0.0282
0.0287

$\mathrm{t}$
0.0000

$-3.2470$

$-0.0932$

0.0287

6.7315

0.1910

0.0284

0.0283

0.0287

1.3755

$-20.1874$

\section{$p$
1.0000
0.0012}

0.0012

0.0000

0.1694

0.0000

LLCI

$-0.0553$

$-0.1496$

0.1351

$-0.0166$

$-0.6351$

ULCI

0.0537

$-0.0235$

0.1042

$-0.1571$

0.2198

0.1133

$-0.4328$
ULCI

0.0553

$-0.0369$

0.2468

0.0946

$-0.5225$

\section{Indirect effects}

total

stereotype awareness $\rightarrow$ stereotype agreement $\rightarrow$ self-esteem

stereotype awareness $\rightarrow$ self-concurrence $\rightarrow$ self-esteem

stereotype awareness $\rightarrow$ stereotype agreement $\rightarrow$ self-concurrence $\rightarrow$ self-esteem

BootSE

Analysis notes:

Level of confidence for all confidence intervals in output: 95.0000

Number of bootstrap samples for percentile bootstrap confidence intervals: 10000

$\begin{array}{rrrr}-0.0140 & 0.0090 & -0.0322 & 0.0032 \\ 0.0073 & 0.0067 & -0.0047 & 0.0218 \\ 0.0000 & 0.0067 & -0.0135 & 0.0132 \\ -0.0213 & 0.0060 & -0.0345 & -0.0108\end{array}$

